\title{
A Self-Calibration Method of Zooming Camera
}

\author{
Ismail El batteoui ${ }^{1}$, Abderrahim Saaidi $^{1,2}$, and Khalid Satori ${ }^{1}$ \\ ${ }^{1}$ LIIAN, Department of Mathematics and Informatics, Faculty of sciences Dhar-Mahraz B.P1796 Atlas Fez, \\ University of Sidi Mohamed Ben Abdellah- Morocco \\ ${ }^{2}$ LSI, Department of Mathematics, Physics and Informatics, Polydisciplinary faculty. B.P 1223, Taza \\ University of Sidi Mohamed Ben Abdellah - Morocco
}

\begin{abstract}
In this article we proposed a novel approach to selfcalibrate a camera with variable focal length. We show that the estimation of camera's intrinsic parameters is possible from only two points of an unknown planar scene. The projection of these points by using the projection matrices in two images only permit us to obtain a system of equations according to the camera's intrinsic parameters. From this system we formulated a nonlinear cost function which its minimization allows us to estimate the camera's intrinsic parameters in each view. The results on synthetic and real data justify the robustness of our method in term of reliability and convergence.
\end{abstract}

Keywords - Variable Focal Length, Unknown Planar Scene, Projection Matrices, Nonlinear Cost Function.

\section{INTRODUCTION}

$\mathrm{T}$ HE self-calibration of camera $[1,2,3,4,5,6,7,8,9,10,11,12,13,1$ $4,15,16]$ is a fundamental area in computer vision, it consist of estimation of camera's parameters with no knowledge about the observed scene. The principle of self-calibration consist to formulate equations based on the camera's intrinsic parameters by using invariant primitives (circular points, absolute conic...) in the scene space. These primitives describe the euclidean structure of the scene.

Our self-calibration method presented in this paper consist of calibrate a camera from a unknown planar scene and by considering that the camera's intrinsic parameters are constants except to the focal length which may be variable from one view to another which is suitable with the current applications of computer vision. The highlight of our idea lies partly in the use of only two points from an unknown plananar scene and the projection of this scene in only two image planes and secondly in the implementation of a relationship between images of the absolute conic in the both views and the projection matrices, to determinate, of the two points used in the scene. The procedure of our method of self-calibration described in this work can be presented in the form of the following steps:

- Acquisition of two images of an unknown planar scene.

- Detection of interest points in the two images by using the Harris detector.

- The matching of the interest points by using the ZNCC correlation measure

- Estimation of the projection matrices of the two points of the scene

- Development of a relationship between the projection matrices estimated in the previous step and the images of the absolute conic in the two images of the scene.

- Formulation of a non-linear cost function on the basis of the relation found in the previous step.

- Optimization of the cost function by the Levenberg-Marquardt
[17] through two phases:

- Initialization phase providing an initial solution by applying constraints on the two images and some intrinsic parameters of the camera.

- Minimizing of the cost function to find the optimum solution according to the intrinsic parameters of our camera in the two images of the scene.

The present article is organized as follows: the second part has a review of previous works dealing with the problem of self-calibration. The self-calibration tools used in this article are discussed in the third section then our method of self-calibration is addressed in the fourth section. Our experimental results are presented in the fifth section and the last part contains a conclusion of our work.

\section{RELATED WORKS}

In this section we present with a non-exhaustive manner some works done in the field of the camera self-calibration. In [18] the singular value decomposition of the fundamental matrix gives a simple form of Kruppa equations that solves the problem of self-calibration of cameras with varying intrinsic parameters. A new method for camera self-calibration performing a sequence of critical motions is processed in [19]. In this method, the intrinsic parameters are assumed to be known except to the focal length which may vary freely from one view to another. The determination of the intrinsic parameters is based on the use of the image of the absolute conic in the different views. In [20], the authors are proposed a new method of self-calibration from a sequence of images of an unknown 3D scene. The projection of the scene points in the image planes is used with the fundamental matrix to determine the projection matrices of scene points. These projection matrices are used to formulate a nonlinear cost function which resolution can estimate the intrinsic parameters in the different views. In [21], the resolution of three linear equations obtained from circles and their centers respectively determine the vanishing line. This vanishing line and the circular points are used to estimate the camera's intrinsic parameters. The problem addressed in [22] is the self-calibration of the cameras with varying intrinsic parameters, This method is based on a quasi-affine reconstruction (by localization the plane in infinity ) which provides a system of equations based on the intrinsic parameters of the camera. The resolution of this system gives the intrinsic parameters in each view. In [23] the authors have proposed a linear approach to self-calibrate a camera performing a small rotation and general translation with the possibility of variation of the focal length. This method is based on using the homography induced by the plane at infinity and the absolute quadric. In [24], a method of camera self-calibration with varying intrinsic parameters is presented. It's based on the quasi-affine reconstruction, after thissreconstruction the homography of the plane at infinity is determined. This homography is used with constraints on the image of the absolute conic to estimate the intrinsic parameters of the camera [29]. 


\section{CAMERA MODEL AND IMAGE OF ABSOLUTE CONIC}

Our camera is modeled by a model called pinhole. Denote by $M=(X Y Z w)^{T}$ a point in homogeneous coordinates of the scene, $m=\left(\begin{array}{llll}x & y & z & w\end{array}\right)^{T}$ an image point. The projection of the $M$ in its image $m$ is denoted by:

$$
m \sim \xi\left(\begin{array}{ll}
R & t
\end{array}\right) M
$$

With $R$ is the rotation matrix and $t$ the translation vector of the camera in space and $\xi$ is the matrix of the intrinsic camera parameters defined by:

$$
\xi=\left(\begin{array}{lll}
l & \gamma & \mu_{0} \\
0 & \eta l & v_{0} \\
0 & 0 & 1
\end{array}\right)
$$

$l:$ Focal length.

$\eta:$ Scale factor.

$\gamma:$ Skew factor.

$\mu_{0}$ and $\nu_{0}$ : Coordinates of principal point.

Considering the space of our scene is a projective space. In this space the points satisfying $w=0$ are called the points at infinity, these points form the plane at infinity. In the plane at infinity the points satisfying $M^{T} M=0$ form the absolute conic denoted $\Omega_{\infty}$. The absolute conic is a complex conic of the plane at infinity, the image of absolute conic $\omega$ is given by:

$$
\omega=\left(\xi \xi^{T}\right)^{-1}
$$

From (3) we deduce that the image of the absolute conic depends only on the intrinsic parameters of the camera. Once $\omega$ is estimated we can easily estimate the intrinsic parameters of the camera using the Cholesky factorization.

\section{PROPOSED METHOD}

The main idea of our new self-calibration method is based on the projection of two points of an unknown planar scene in only two image planes using two projection matrices which must be estimated.

Let $(S)$ an unknown planar scene and $A\left(x_{A}, y_{A}, z_{A}, 1\right)^{T}$ and $B\left(x_{B}, y_{B}, z_{B}, 1\right)^{T}$ two points in homogeneous coordinates of $(S)$ and $a_{k}\left(u_{a k}, v_{a k}, 1\right)^{T}$ and $b_{k}\left(u_{b k}, v_{b k}, 1\right)^{T}$ for $k=i$ or $j$ its projections in the two images (see Fig. 1).

Assuming that the plane containing the segment $[A B]$ is in $Z=0$ and $0<\theta<\Pi / 2$. From the Fig. 1 we can deduce: $\quad x_{A}=\frac{\|A B\|}{2} \cos (\theta) \quad$ and $\quad y_{A}=\frac{\|A B\|}{2} \sin (\theta)$
$x_{B}=-\frac{\|A B\|}{2} \cos (\theta)$ and $y_{B}=-\frac{\|A B\|}{2} \sin (\theta)$

From (1) we have for the image i :

$$
a_{i} \sim \xi_{i}\left(\begin{array}{llll}
r_{1 i} & r_{2 i} & r_{3 i} & t_{i}
\end{array}\right) A
$$

with $r_{q i}$ is the column number $q$ of $R_{i}$.

The equation (4) can be rewritten as follows:

$$
a_{i} \sim \xi_{i}\left(\begin{array}{lll}
r_{1 i} & r_{2 i} & t_{i}
\end{array}\right)\left(\begin{array}{l}
x_{A} \\
y_{A} \\
1
\end{array}\right)
$$

Let:

$$
H_{i} \sim \xi_{i}\left(r_{1 i} r_{2 i} t_{i}\right)
$$

$H_{i}$ is a $3 \times 3$ matrix called the homography matrix scene to image $i$. The equation (5) becomes:

$$
a_{i} \sim H_{i}\left(\begin{array}{l}
x_{A} \\
y_{A} \\
1
\end{array}\right)
$$

Let $H_{i}=\left(h_{1 i} h_{2 i} h_{3 i}\right)$ with $h_{k i}$ are column vectors of $H_{i}$. The vectors $r_{1 i}$ and $r_{2 i}$ are orthogonal which permit us to write from (6) :

$$
\left\{\begin{array}{l}
h_{1 i}^{T} \xi_{i}^{-T} \xi_{i}^{-1} h_{2 i}=0 \\
h_{1 i}^{T} \xi_{i}^{-T} \xi_{i}^{-1} h_{1 i}=h_{2 i}^{T} \xi_{i}^{-T} \xi_{i}^{-1} h_{2 i}
\end{array}\right.
$$



Fig. 1. Vision system used

Let:

$$
A^{\prime}=\left(\begin{array}{lll}
x_{A} & y_{B} & 1
\end{array}\right)^{T} \text { and } B^{\prime}=\left(\begin{array}{lll}
x_{B} & y_{B} & 1
\end{array}\right)^{T}
$$


From (7) we have for image $i$ :

$$
\left\{\begin{array}{l}
a_{i} \sim H_{i} A^{\prime} \\
b_{i} \sim H_{i} B^{\prime}
\end{array}\right.
$$

For image $j$ :

$$
\left\{\begin{array}{l}
a_{j} \sim H_{j} A^{\prime} \\
b_{j} \sim H_{j} B^{\prime}
\end{array}\right.
$$

From (9) and (10) we can write:

$$
\left\{\begin{array}{l}
a_{i} \sim H_{i} Q W \\
a_{j} \sim H_{j} Q W
\end{array}\right.
$$

And:

$$
\begin{aligned}
& \left\{\begin{array}{l}
b_{i} \sim H_{i} Q W^{\prime} \\
b_{j} \sim H_{j} Q W^{\prime}
\end{array}\right. \\
& Q=\left(\begin{array}{ccc}
\frac{\|A B\|}{2} \cos (\theta) & 0 & 0 \\
0 & \frac{\|A B\|}{2} \sin (\theta) & 0 \\
0 & 0 & 1
\end{array}\right), W=\left(\begin{array}{l}
1 \\
1 \\
1
\end{array}\right) \text { and } W^{\prime}=\left(\begin{array}{r}
-1 \\
-1 \\
1
\end{array}\right) .
\end{aligned}
$$

Let:

$$
\left\{\begin{array}{l}
P_{i}=H_{i} Q \\
P_{j}=H_{j} Q
\end{array}\right.
$$

The equations (11) and (12) become:

$$
\left\{\begin{array}{l}
a_{i} \sim P_{i} W \\
a_{j} \sim P_{j} W
\end{array}\right.
$$

And:

$$
\left\{\begin{array}{l}
b_{i} \sim P_{i} W^{\prime} \\
b_{j} \sim P_{j} W^{\prime}
\end{array}\right.
$$

The matrices $P_{i}$ and $P_{j}$ are the projection matrices of the points $A$ and $B$ in the both images $i$ and $j$.

From (13) we can write:

$$
P_{j}=H_{i j} P_{i}
$$

With $H_{i j}=H_{j} H_{i}^{-1}$ is the homography matrix between the images $i$ and $j$. From (14), (15) and (16) we can write:

$$
\left\{\begin{array}{l}
a_{i} \sim P_{i} W \\
a_{j} \sim H_{i j} P_{i} W \\
b_{i} \sim P_{i} W^{\prime} \\
b_{j} \sim H_{i j} P_{i} W^{\prime}
\end{array}\right.
$$

The matrix $P_{i}$ consists of eight unknowns and the above system gives us twelve equations that allows us to estimate the matrix $P_{i}$. The matrix $P_{j}$ can be estimated from (16).

Let $P_{i}=\left(\begin{array}{lll}p_{1 i} & p_{2 i} & p_{3 i}\end{array}\right)$, with the $p_{k i}$ are the column vectors of $P_{i}$. From (13) we can deduce:

$$
\left\{\begin{array}{l}
p_{1 i}=\frac{\|A B\|}{2} \cos (\theta) h_{1 i} \\
p_{2 i}=\frac{\|A B\|}{2} \sin (\theta) h_{2 i}
\end{array}\right.
$$

The system of equations (18) gives us:

$$
\left\{\begin{array}{l}
h_{1 i}=\frac{2}{\|A B\|} \cos (\theta)^{-1} p_{1 i} \\
h_{2 i}=\frac{2}{\|A B\|} \sin (\theta)^{-1} p_{2 i}
\end{array}\right.
$$

By replacing in (9) $h_{1 i}$ and $h_{2 i}$ by their expressions in (19) and by posing $\lambda_{1}=\frac{2}{\|A B\|} \cos (\theta)^{-1}$ and $\lambda_{2}=\frac{2}{\|A B\|} \sin (\theta)^{-1}$, we obtain for image $i$ :

$$
\left\{\begin{array}{l}
\lambda_{1} \lambda_{2} p_{1 i}^{T} \omega_{i} p_{2 i}=0 \\
\lambda_{1}^{2} p_{1 i}^{T} \omega_{i} p_{1 i}=\lambda_{2}^{2} p_{2 i}^{T} \omega_{i} p_{2 i}
\end{array}\right.
$$

Or:

$$
\left\{\begin{array}{l}
p_{1 i}^{T} \omega_{i} p_{2 i}=0 \\
\frac{\lambda_{1}^{2}}{\lambda_{2}^{2}}=\frac{p_{2 i}^{T} \omega_{i} p_{2 i}}{p_{1 i}^{T} \omega_{i} p_{1 i}}
\end{array}\right.
$$

And for image $j$ :

$$
\left\{\begin{array}{l}
\lambda_{1} \lambda_{2} p_{1 j}^{T} \omega_{j} p_{2 j}=0 \\
\lambda_{1}^{2} p_{1 j}^{T} \omega_{j} p_{1 j}=\lambda_{2}^{2} p_{2 j}^{T} \omega_{j} p_{2 j}
\end{array}\right.
$$

Or:

$$
\left\{\begin{array}{l}
p_{1 j}^{T} \omega_{j} p_{2 j}=0 \\
\frac{\lambda_{1}^{2}}{\lambda_{2}^{2}}=\frac{p_{2 j}^{T} \omega_{j} p_{2 j}}{p_{1 j}^{T} \omega_{j} p_{1 j}}
\end{array}\right.
$$

From (20) and (21) we can deduce that:

$$
\frac{p_{2 i}^{T} \omega_{i} p_{2 i}}{p_{1 i}^{T} \omega_{i} p_{1 i}}=\frac{p_{2 j}^{T} \omega_{j} p_{2 j}}{p_{1 j}^{T} \omega_{j} p_{1 j}}
$$


Or:

$$
p_{2 i}^{T} \omega_{i} p_{2 i} p_{1 j}^{T} \omega_{j} p_{1 j}=p_{2 j}^{T} \omega_{j} p_{2 j} p_{1 i}^{T} \omega_{i} p_{1 i}
$$

Let $H_{i j}=\left(\begin{array}{l}\bar{h}_{i j 1} \\ \bar{h}_{i j 2} \\ \bar{h}_{i j 3}\end{array}\right)$ with $\bar{h}_{i j 1}, \bar{h}_{i j 2}$ and $\bar{h}_{i j 3}$ are the row vectors of $H_{i j}$.

From (16) we can deduce:

$$
\left\{\begin{array}{l}
p_{1 j}=\bar{h}_{i j 1} p_{1 i} \\
p_{2 j}=\bar{h}_{i j 2} p_{2 i} \\
p_{3 j}=\bar{h}_{i j 3} p_{3 i}
\end{array}\right.
$$

By replacing $p_{1 j}$ and $p_{2 j}$ by its expressions in (22) we obtain:

$$
p_{2 i}^{T} \omega_{i} p_{2 i}\left(\bar{h}_{i j 1} p_{1 i}\right)^{T} \omega_{j}\left(\bar{h}_{i j 1} p_{1 i}\right)=\left(\bar{h}_{i j 2} p_{2 i}\right)^{T} \omega_{j}\left(\bar{h}_{i j 2} p_{2 i}\right) p_{1 i}^{T} \omega_{i} p_{1 i}
$$

By developing the first equation in (13) we obtain for image $i$ :

$$
P_{i}^{T} \omega_{i} P_{i}=\left(\begin{array}{cc}
Q^{\prime T} Q^{\prime} & Q^{\prime T} R_{i}^{T} t_{i} \\
t_{i}^{T} R_{i} Q^{\prime} & t_{i}^{T} t_{i}
\end{array}\right)
$$

With $\omega_{i}=\left(\xi_{i} \xi_{i}^{T}\right)^{-1}$ is the image of absolute conic in image $i$ and

$$
Q^{\prime}=\left(\begin{array}{cc}
\frac{\|A B\|}{2} \cos (\theta) & 0 \\
0 & \frac{\|A B\|}{2} \cos (\theta) \\
0 & 0
\end{array}\right)
$$

For the image $j$ :

$$
P_{j}^{T} \omega_{j} P_{j}=\left(\begin{array}{cc}
Q^{\prime} Q^{\prime} & Q^{\prime T} R_{j}^{T} t_{j} \\
t_{j}^{T} R_{j} Q^{\prime} & t_{j}^{T} t_{j}
\end{array}\right)
$$

Let:

$$
P_{i}^{T} \omega_{i} P_{i}=\left(\begin{array}{lll}
e_{11 i} & e_{12 i} & e_{13 i} \\
e_{21 i} & e_{22 i} & e_{23 i} \\
e_{31 i} & e_{32 i} & e_{33 i}
\end{array}\right)
$$

and:

$$
P_{j}^{T} \omega_{j} P_{j}=\left(\begin{array}{lll}
e_{11 j} & e_{12 j} & e_{13 j} \\
e_{21 j} & e_{22 j} & e_{23 j} \\
e_{31 j} & e_{32 j} & e_{33 j}
\end{array}\right)
$$

From (25) and (26) we can deduced that the first two rows and columns of the matrices $P_{i}^{T} \omega_{i} P_{i}$ and $P_{j}^{T} \omega_{j} P_{j}$ are identical, which permit us to write:

$$
\left\{\begin{array}{l}
e_{11 i} e_{12 j}-e_{11 j} e_{12 i}=0 \\
e_{11 i} e_{22 j}-e_{11 j} e_{22 i}=0
\end{array}\right.
$$

From (20), (21), (22), (24) and (29) we can deduce the following system:

$$
\left\{\begin{array}{l}
p_{1 i}^{T} \omega_{i} p_{2 i}=0 \\
p_{1 j}^{T} \omega_{j} p_{2 j}=0 \\
p_{2 i}^{T} \omega_{i} p_{2 i} p_{1 j}^{T} \omega_{j} p_{1 j}=p_{2 j}^{T} \omega_{j} p_{2 j} p_{1 i}^{T} \omega_{i} p_{1 i} \\
p_{2 i}^{T} \omega_{i} p_{2 i}\left(\bar{h}_{i j 1} p_{1 i}\right)^{T} \omega_{j}\left(\bar{h}_{i j 1} p_{1 i}\right)=\left(\bar{h}_{i j 2} p_{2 i}\right)^{T} \omega_{j}\left(\bar{h}_{i j 2} p_{2 i}\right) p_{1 i}^{T} \omega_{i} p_{1 i} \\
e_{11 i} e_{12 j}-e_{11 j} e_{12 i}=0 \\
e_{11 i} e_{22 j}-e_{11 j} e_{22 i}=0
\end{array}\right.
$$

In the present article we are assumed in the one hand that the intrinsic camera parameters are unknown and constant except for the focal length which can be vary from one view to another and in the second hand we are used two images only of an unknown planar scene which gives us six unknown, the system of equations (30) contains six equations where the possibility to estimate the intrinsic parameters of camera in the both views.

The equations of the system (30) are nonlinear therefore to solve it we minimize the cost function given by the following expression:

$$
\min _{\Delta_{i j}} \sum_{j=i+1}^{n} \sum_{i=1}^{n-1}\left(\beta_{1 i}^{2}+\beta_{2 j}^{2}+\beta_{3 i j}^{2}+\beta_{4 i j}^{2}+\beta_{5 i j}^{2}+\beta_{6 i j}^{2}\right)
$$

with:

$$
\begin{aligned}
& \beta_{1 i}=p_{1 i}^{T} \omega_{i} p_{2 i} \\
& \beta_{2 j}=p_{1 j}^{T} \omega_{j} p_{2 j} \\
& \beta_{3 i j}=p_{2 i}^{T} \omega_{i} p_{2 i} p_{1 j}^{T} \omega_{j} p_{1 j}-p_{2 j}^{T} \omega_{j} p_{2 j} p_{1 i}^{T} \omega_{i} p_{1 i} \\
& \beta_{4 i j}=p_{2 i}^{T} \omega_{i} p_{2 i}\left(\bar{h}_{i j 1} p_{1 i}\right)^{T} \omega_{j}\left(\bar{h}_{i j 1} p_{1 i}\right)-\left(\bar{h}_{i j 2} p_{2 i}\right)^{T} \omega_{j}\left(\bar{h}_{i j 2} p_{2 i}\right) p_{1 i}^{T} \omega_{i} p_{1 i} \\
& \beta_{5 i j}=e_{11 i} e_{12 j}-e_{11 j} e_{12 i} \\
& \beta_{6 i j}=e_{11 i} e_{22 j}-e_{11 j} e_{22 i}
\end{aligned}
$$

And $\Delta_{i j}$ is the vector of the intrinsic parameters of the camera in the two images $i$ and $j$ and $n$ is the number of images used.

The minimization of (31) is given by the Levenberg-Marquardt algorithm which requires an initialization step, for this purpose we assume the following assumptions:

- The main point is in the center of the image, so $\mu_{i 0}, v_{i 0}, \mu_{j 0}$ and $v_{j 0}$ are known.

- The pixels are square so $\eta_{i}=\eta_{j}=1$ and $\gamma_{i}=\gamma_{j}=0$.

By substituting these parameters in the system of equations (30) we can determine the focal lengths $l_{i}$ and $l_{j}$ for the both images $i$ and $j$. 


\section{EXPERIMENTAL RESULTS}

To test the effectiveness of our method two types of data are used synthetic and real.

\section{A. Simulation}

In this simulation a sequence of ten $512 \times 512$ images of a calibration target is used to test the effectiveness of our self-calibration method presented in this paper. After the detection of interest points by the Harris detector [25] the corresponding points between images are determined using the correlation measure ZNCC [26]. The images are perturbed by adding a Gaussian noise of deviation $\sigma$ to the pixels of images. The projection of the scene in image planes allows us to obtain linear equations whose its solution allows to determine the projection matrices. The self-calibration equations are then built to formulate a nonlinear cost function which its minimization by the Levenberg-Marquardt algorithm helps us to estimate the intrinsic parameters of our camera in all views. To evaluate the performance of our approach we compared the results obtained with those given by a well-known calibration method [6] published by Z. Zhang and those given by [27]. The figures (2) and (3) consecutively present the relative error of the focal length estimated by our method and those of Z. Zhang and C-R.Huang according to the number of images used and added noise.



Fig. 2. Relative error on the focal length according to the number of images used.



Fig. 3. Relative error on the focal length according to the added noise
The both figures above show that the method developed in this paper and that of Z.Zhang gives the approximate results. By taking into account the robustness of the Z. Zhang's method we can say that our method is effective and efficient. On the other hand, the previous figures prove the preference of our method compared with that presented in [27].

\section{B. Real images}

In this second experiment, two $512 \times 512$ images of a real planar scene are acquired by a CCD camera having constant intrinsic parameters except to the focal length. (See Fig. 4).


Fig. 4. The two real images used

The interest points are determined as well as their matching and calculation of projection matrices.

1. Detection of interest points

The interest points in the two images are detected by using the Harris detector (Fig. 5).
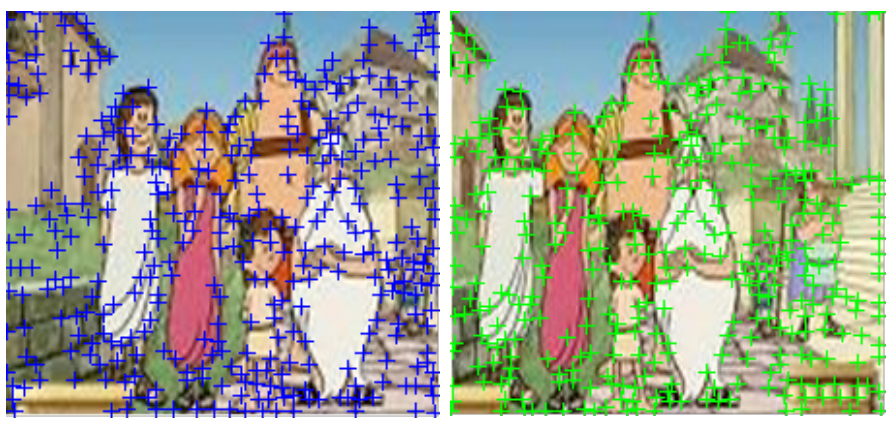

Fig. 5. Detection of interest points

\section{Matching of interest points}

The interest points previously identified are matched between the two images by using the ZNCC algorithm (Fig. 6).


Fig. 6. Matching of interest points.

\section{Estimation of intrinsic parameters}

This step consists of estimating the intrinsic parameters of the camera in each view by applying our method. The following table shows the results obtained. 
TABLE I

THE RESULTS OBTAINED BY OUR METHOD

\begin{tabular}{ccccccc}
\cline { 3 - 7 } & & $l$ & $\eta$ & $\gamma$ & $\mu_{0}$ & $v_{0}$ \\
\hline \multirow{2}{*}{ Our method } & Image 1 & 1687 & 0,95 & 0,09 & 258 & 260 \\
& Image 2 & 1683 & 0,97 & 0,05 & 257 & 255 \\
\hline
\end{tabular}

The above table presents the results obtained by our method of self-calibration discussed in this article. To verify the effeteness of the above results, we rectified the two used images by using a robust approach of image rectification [28] based on a calibrated camera. The following figure shows the rectified images obtained by applying [28] and using the values of table 1 .


Fig.. 7 The obtained rectified images.

The goal of image rectification is to obtain the epipolar lines horizontal and parallel to the x-axis, according to the Fig. 7 we can deduce that our approach presented in this paper gives satisfactory results and this by taking into account that our method is able to selfcalibrate a camera with a variable focal length from two images only based on two points only of an unknown planar scene.

\section{CONCLUSION}

In this work we have proposed a new method of self-calibration of a CCD camera with constant intrinsic parameters except to the focal length which can vary freely from one view to another from an unknown planar scene. We have shown that the determination of the intrinsic parameters is possible by using only two points of the scene. The projections of the two points in the two image planes permit us to determine their projection matrices, these matrices are used to formulate a nonlinear cost function. The minimization of the cost function obtained allowed us to estimate the intrinsic parameters of the camera. The experimental results on the synthetic and real data prove the robustness of our method.

\section{REFERENCES}

[1] B. Triggs. Autocalibration from Planar Scenes. In European Conference on Computer Vision, volume 2, pages 89-105, Friburg, Allemagne, juin 1998.

[2] R. Hartley and A. Zisserman. Multiple View Geometry. Seconde dition. Cambridge University Press, 2003.

[3] M. Merras, N. El Akkad, A. Saaidi and K. Satori. Camera Calibration with Varying Parameters Based On Improved Genetic Algorithm. WSEAS TRANSACTIONS on COMPUTERS. Vol. 13, 2014.

[4] P.Gurdjos, A.Crouzil and R.Payrissat. Another Way of Looking at PlaneBased Calibration:The Centre Circle Constraint.ECCV, 2002.

[5] P.F.Sturm and S.J.Maybank. On Plane-Based Camera Calibration: A General Algorithm, Singularities, Applications. In Proceedings of the CVPR-IEEE, Vol. 1, pp. 432-437, 1999.

[6] Z.Zhang. A Flexible New Technique for camera Calibration. IEEE Transactions on Pattern Analysis and Machine Intelligence, Vol. 22, No.17, pp. 1730-1734, 2000.
[7] M.Wilczkowiak, E.Boyer, P.Sturm. Camera Calibration and 3D Reconstruction from Single Images Using Parallelepipeds.In ICCV, Vancouver, Canada, pp. 162-169, July 2001.

[8] A.Saaidi, A.Halli, H.Tairi and K.Satori. Self-Calibration Using a Particular Motion of Camera. Wseas Transaction on Computer . Issue 4, Vol. 3, April 2008.

[9] P.Gurdjos and P.Sturm. Methods and Geometry for Plane-Based SelfCalibration. CVPR, pp. 491-496, 2003.

[10] B.Triggs. Autocalibration from planar sequences, In Proceedings of 5th EuropeanConference on Computer Vision,Freiburg, Allemagne, Juin 1999.

[11] N. El Akkad, M. Merras, A. Saaidi and K. Satori. Camera self-Calibration with Varying Parameters from Two views. WSEAS TRANSACTIONS on INFORMATION SCIENCE and APPLICATIONS, Issue.11, Vol. 10, 2013.

[12] I.EL Batteoui, A.Saiidi and K.Satori. Efficient planar self-calibration of a stationary camera with variable intrinsic parameters, JATIT, Vol. 50 No.1, April 2013.

[13] A.Hehden and K.Astrom,Euclidean Reconstruction from Images Sequences with Varying and unknown focal length and Principal Point, Proc.IEEE CVPR,pp.438-443,1997.

[14] A. Saaidi, A. Halli, H. Tairi and K. Satori. Self-Calibration Using a Planar Scene and Parallelogram. ICGST-GVIP, ISSN 1697 399X, February 2009.

[15] L.Agapito,E.Hayman and I.Reid. Self-calibration of rotating and zooming camera. In International Journal of Computer Vision,pp.107-127,2001

[16] J. Semple and G. Kneebone. Algebraic Projective Geometry. Oxford Classic Series, Clarendon Press, 1952, réédité, 1998. 2012.

[17] J.More.The levenberg-marquardt Algorithm, implementation and theory. In G.A.Watson, editor, Numerical Analysis, Lecture Notes in Mathematics 630. Springer-Verlag, 1977.

[18] Manolis I.A. Lourakis and R.Deriche. Camera self-calibration using the kruppa equations and the SVD of the fundamental matrix: the case of varying intrinsic parameters. Technical Report 3911, INRIA, 2000.

[19] P.Sturm. Critical motion sequences for the self-calibration of cameras and stereo systems with variable focal length. Image and Vision Computing, 20(5-6) : 415-426, 2002.

[20] N. El Akkad, M. Merras, A.Saaidi and K.Satori, Camera self-calibration with varying intrinsic parameters by an unknown three dimensional scene, Visual Computer, vol.30, No.5, pp.519-530, May 2014.

[21] Y.Zhao and X.D.Lv. A new approach for camera-self-calibration using vanishing-line. Information Technology Journal, 11(2) :276- 282, 2012.

[22] Z.Jiang and S.Liu, The Self-calibration of Varying Internal Camera Parameters Based on Image of Dual Absolute Quadric Transformation, Information and Automation, Communications in Computer and Information Science, Springer, Vol.86, pp. 452-461, 2011.

[23] J.-S.Liu,J.-H, Self-calibration with varying focal length from two images obtained by a camera with small rotation and general translation. Pattern recognition letters- Elsevier 22 (2001) 1393-1404.

[24] Zetao Jiang. Self-calibration of Varying Internal Camera Parameters Algorithm Based on Quasi-affine Reconstruction. Journal of Computers, Vol. 7, No. 3, March 2012.

[25] C.Harris and M.Stephens. A combined Corner and Edge Detector. 4th Alvey vision Conference. Pp. 147-151, 1988.

[26] C.Saravananand, M.Surender. Algorithm for Face Matching Using Normalized Cross-Correlation. IJEAT, Vol.2, Issue 4,April 2013.

[27] C-R. Huang, C-S. Chen, and P-C. Chung, An Improved Algorithm for Two-image Camera Self-Calibration and Euclidean Structure Recovery Using Absolute Quadric, Pattern Recognition, vol. 37, no. 8, pp. 17131722, 2004.

[28] Fusiello, A., Trucco, E., \& Verri, A. A compact algorithm for rectification of stereo pairs. Machine Vision and Applications, 12(1), 16-22, 2000.

[29] Pacheco, A., H. Bolivar-Baron, R. Gonzalez-Crespo, and J. PascualEspada, Reconstruction of High Resolution 3D Objects from Incomplete Images and 3D Information. International Journal of Interactive Multimedia and Artificial Intelligence, 6(2), 7-16, 2014. 




Ismail El batteoui received the master's degree from SMBA-Fez University in 2010. He is currently working toward the PhD degree in the LIIAN Laboratory at SMBA Fez University. His current research interests include camera self calibration with variable intrinsic parameters, image rectification and $3 \mathrm{D}$ reconstruction.

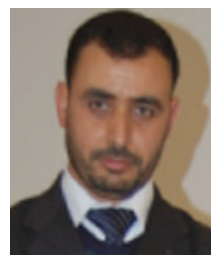

Abderrahim Saaidi received the $\mathrm{PhD}$ degree from SMBA-Fez University in 2010. He is currently a professor of computer science at SMBA-Taza University. He is a member of the LIIAN and LSI Laboratories. His research interests include camera self calibration, 3D reconstruction, genetic algorithms and real-time rendering.



Khalid Satori received the $\mathrm{PhD}$ degree from the National Institute for the Applied Sciences INSA at Lyon in 1993. $\mathrm{He}$ is currently a professor of computer science at SMBAFez University. He is the director of the LIIAN Laboratory. His research interests include real-time rendering, Imagebased rendering, virtual reality, biomedical signal, camera self calibration, genetic algorithms and $3 \mathrm{D}$ reconstruction. 